\title{
Mischungsentropie und osmotischer Druck von Lösungen langgestreckter Teilchen mit innerer Beweglichkeit (Zur statistischen Theorie makromolekularer Lösungen III ${ }^{1}$ )
}

\author{
Von G. V. Schulz \\ Aus dem Institut für physikalische Chemie der Universität Mainz \\ (Z. Naturforschg. 2 a, 411-419 [1947]; eingegangen am 11. Februar 1947)
}

\begin{abstract}
Die Mischungsentropie der Lösungen von Fadenmolekülen mit innerer Beweglichkeit wird auf molekularstatistischem Weg abgeleitet. Der Rechnung wird ein Molekülmodell zugrundegelegt, das aus einer Anzahl starrer, gegeneinander vollständig frei beweglicher Glieder (Segmente) in linearer Anordnung besteht. Je beweglicher ein Fadenmolekül ist, um so mehr Segmente besitzt das ihm zugeordnete Modellmolekül.

Die Rechnung ergibt, daß die Mischungsentropie und damit auch der osmotische Druck höher ist, als dem Idealgesetz entspricht, und daß diese Erhöhung von dem Verhältnis der Länge zum Durchmesser des einzelnen Segmentes bestimmt wird. Fadenmoleküle mit völlig unbehinderter freier Drehbarkeit der Kettenglieder ergeben eine ,ideale“ Mischungsentropie, und ihr osmotischer Druck gehorcht dem van t'Hoff schen Gesetz, wenn ihre Verdünnungswärme gleich Null ist.

In der Literatur findet sich bisher nur ein Beispiel einer athermischen Lösung eines hochpolymeren Stoffes, bei welcher der osmotische Druck, die Verdünnungswärme und die Entropie experimentell bestimmt sind (Guttapercha in Toluol). An dieser ergibt sich die Segmentlänge nach osmotischen Messungen als ziemlich gut übereinstimmend mit der auf viskosimetrischem Wege ermittelten (W. K u h n). Bei Lösungen mit endlichen (positiven oder negativen) Werten der Verdünnungswärme ist, wie gezeigt wird, die experimentell bestimmte Verdünnungsentropie nicht identisch mit dem Mischungsanteil der Verdünnungsentropie, so daß man aus osmotischen Messungen an solchen Lösungen bisher keinen Anhalt über die Gestalt der Moleküle gewinnen kann.
\end{abstract}

$I^{\prime \prime}$ $\mathrm{n}$ der zweiten Arbeit dieser Reihe ${ }^{1}$ wurde eine Gleichung für die Mischungsentropie und den osmotischen Druck von Lösungen zylinderischer Teilchen aufgestellt. Diese ließ sich an Proteinlösungen bestätigen, für welche die bei der Ableitung gemachten Voraussetzungen, daß die Teilchen starr sind und daß ihre Verdünnungswärme bei kleinen Konzentrationen vernachlässigt werden kann, als zutreffend anzusehen ist.

Um auch den osmotischen Druck von Lösungen fadenförmiger Moleküle mit mehr oder weniger großer innerer Beweglichkeit zu beherrschen, ist es notwendig, die Rechnung unter Einführung neuer Voraussetzungen durchzuführen. Es liegen bereits eine Reihe von Arbeiten verschiedener Autoren ${ }^{2,3,4}$ hierüber vor, deren Ergebnisse z. Tl. von einander abweichen (vergl. die Einleitung zur Arbeit $\mathrm{II}^{1}$ ). Da die vom Verfasser angewandte Rechnungsart von derjenigen der anderen

1 I. Mitteilung: G. V. Sch u l z, Z. Naturforschg. 2 a, 27 [1947]; II. Mitteilung: ebenda 2a, 348 [1947].

2 P. J. F l o r y, J. Chem. Physics 9, 660 [1941]; 10, 51 [1942].
Autoren abweicht und einige etwas zweifelhafte Voraussetzungen vermeidet, schien es zweckmäßig, sie auch auf innerlich bewegliche Kettenmoleküle zu erweitern. Wir legen der Rechnung ein Modell zugrunde, das es in einfacher Weise erlaubt, das Ergebnis osmotischer Messungen mit demjenigen anderer Messungen (etwa der Viscosität oder der Strömungsdoppelbrechung) zu vergleichen.

$\mathrm{Da}$ die Rechnungen der vorliegenden Arbeit auf denen der vorangegangenen Arbeiten ${ }^{1}$ basieren, werden wir gelegentlich Gleichungen aus diesen verwenden. Wir zitieren sie, indem wir mit römischen Ziffern die Arbeit, mit arabischen die Gleichung bezeichnen: also (II, 27) bedeutet Gleichung (27) aus der II. Mitteilung. In gleicher Weise werden die Tabellen und Abbildungen angeführt.

\section{A n s a tz}

Nach W. K u h n ${ }^{5}$ kann man ein Fadenmolekül, dessen Kettenglieder gegeneinander mehr oder

3 M. L. H u g g in s, J. Physic. Chem. 9, 440 [1941].

${ }_{4}$ A. M ü n s t e r, Kolloid. Z. 105, 1 [1943]; Z. Naturforschg. 1, 311 [1946].

${ }_{5}$ W. K u hn, Kolloid. Z. 68, 2 [1934]; Angew. Chem. 49, 858 [1936]. 
weniger beweglich sind, durch ein Modell ersetzen, das aus einer Anzahl von starren „Segmenten“ besteht, die vollständig frei gegeneinander beweglich sind. Die Größe eines Segments (nach K u h n eines statistischen Fadenelements) ist folgendermaßen definiert: Gehen wir von einem Grundmolekül zum nächsten über, so wird dessen Achse im allgemeinen gegen diejenige des ersten geneigt sein: es wird mehrere Raumorientierungen einnehmen können, jedoch nicht alle überhaupt denkbaren, da die Drehbarkeit um die Valenzachse meist mehr oder weniger behindert ist. Gehen wir nun zum übernächsten Grundmolekül über und so fort, so werden wir nach einer Anzahl von $y$ Schritten ein Grundmolekül erreichen, dessen Orientierung nicht mehr von der Orientierung des ersten Grundmoleküls abhängt. Dann ist definitionsgemäß $y$ die Anzahl der Grundmoleküle, aus denen sich ein Segment zusammensetzt.

Das gesamte Fadenmolekül können wir uns als eine Kette solcher Segmente vorstellen, die in sich starr, aber gegeneinander völlig frei beweglich sind. Je beweglicher ein Fadenmolekül ist, aus um so mehr derartigen Segmenten ist es zusammengesetzt. Ein völlig starres Molekül dagegen besteht nur aus einem Segment. Die Länge der Segmente ist also ein Maß für die Starrheit des Fadenmoleküls.

Zur Berechnung der thermodynamischen Wahrscheinlichkeit einer Mischung aus $N_{1}$ Lösungsmolekülen und $N_{2}$ Makromolekülen, deren jedes aus $x$ Segmenten besteht, machen wir eine analoge Betrachtung wie in der II. Mitteilung. Hierzu führen wir einige zusätzliche Bezeichnungen ein:

Die Zahl der Gitterpunkte, die ein Makromolekül besetzt, sei wieder

$$
n=v_{2} / v_{1},
$$

wobei $v_{2}$ und $v_{1}$ die Molvolumina der beiden Komponenten sind. Die Zahl der Gitterpunkte, die ein Makromolekül blockiert (innerer Hof), sei $x n$, wobei $x$ wie in der vorigen Arbeit zu berechnen ist.

Der ,äußere Hof“ (vergl. II, Abb.1) ist dagegen für ein bewegliches Molekül anders zu berechnen wie für ein starres. Da jedes Segment völlig unabhängig von seinen Nachbarsegmenten drehbar ist, ist der Raum, in welchem eine Behinderung der Orientierung eintritt, nur durch das Achsenverhältnis der Segmente bestimmt. Bezeichnen wir die Zahl der im äußeren Hof eines Segmentes liegenden Gitterpunkte mit $n_{a s}$, so bedeckt der äußere Hof eines ganzen Moleküls

\section{Gitterpunkte.}

$$
n_{a}=x n_{a s}
$$

Befindet sich ein Segment im äußeren Hof eines anderen, so sind seine räumlichen Orientierungsmöglichkeiten eingeschränkt. Hat ein unbehindertes Segment $z$ Orientierungsmöglichkeiten, so besitzt ein im äußeren Hof eines anderen befindliches im Durchschnitt nur $z\left(1-\bar{\alpha}_{s}\right)$ Orientierungsmöglichkeiten. Dabei hängt $\bar{\alpha}_{s}$ vom Achsenverhältnis eines Segmentes ab.

\section{Die Anzahl der Anordnungs- möglichkeiten}

Die Lösung hat $N_{1}+n N_{2}$ Gitterpunkte. Auf diese sind $N_{2}$ Makromoleküle zu verteilen, wobei die verschiedenen Einstellmöglichkeiten in Rechnung zu setzen sind. Wir gehen wieder nach der "Auffüllmethode" vor und bestimmen zunächst die Zahl der Anordnungen für das

\section{Molekül.}

Dessen 1. Segment stehen $N_{1}+n N_{2}$ Plätze zur Verfügung; auf jedem hat es $z$ mögliche räumliche Orientierungen. Das 1. Segment hat also $z\left(N_{1}+n N_{2}\right) \quad$ Anordnungsmöglichkeiten. Das 2. Segment habe $z^{\prime}$ Orientierungen ${ }^{6}$, und ebenso jedes weitere. Das 1. Makromolekül hat also im ganzen

$$
z\left(N_{1}+n N_{2}\right) \cdot z^{\prime(x-1)}
$$

Anordnungsmöglichkeiten.

Entscheidend für die weitere Rechnung ist die Beurteilung der Anordnungsmöglichkeiten für das

\section{Molekül.}

Dessen 1. Segment stehen insgesamt $N_{1}+n N_{2}$ $-n_{a}-x_{n}$ unbehinderte Plätze zur Verfügung, auf welchen es $z$ verschiedene Einstellungen haben kann. Hinzu kommen $n_{a}$ Plätze mit $z\left(1-\bar{\alpha}_{s}\right)$ Orientierungsmöglichkeiten. Es hat also

$$
z\left(N_{1}+n N_{2}-n_{a}-x n\right)+z n_{a}\left(1-\bar{a}_{s}\right)
$$

${ }^{6}$ Man kann annehmen, daß $z^{\prime}=z-1$ ist. Aber auch eine stärkere Beschränkung wäre denkbar. Indessen hat das keinen Einfluß auf das hier behandelte Problem. 
Anordnungsmöglichkeiten. Führen wir noch ein:

$$
\beta_{s} \equiv \bar{a}_{s} n_{a}+\varkappa n,
$$

so erhalten wir für das 1. Segment des 2. Moleküls $z\left(N_{1}+n N_{2}-\beta\right)$ Anordnungsmöglichkeiten.

Der Anfangspunkt* des 2.Segments kann entweder auf einem unbehinderten Platz stehen, dann hat es $z^{\prime}$ Einstellmöglichkeiten; oder im Behinderungsraum eines anderen Moleküls, dann hat es $z^{\prime}\left(1-\bar{\alpha}_{s}\right)$ Einstellmöglichkeiten. Es existieren nun $\mathrm{N}_{1}+n N_{2}-n_{\alpha}-x n$ unbehinderte Plätze und $n_{a}$ behinderte Plätze. Die Wahrscheinlichkeit dafür, daß es sich auf einem unbehinderten Platz befindet, ist $\frac{N_{1}+n N_{2}-n_{a}-\varkappa n}{N_{1}+n N_{2}-\varkappa n}$, daß es sich auf einem behinderten Platz befindet, ist

$$
\frac{n_{a}}{N_{1}+n N_{2}-\varkappa n} \text {. }
$$

Seine gesamten Einstellmöglichkeiten sind also

$$
\begin{aligned}
& z^{\prime} \frac{N_{1}+n N_{2}-n_{a}-\varkappa n}{N_{1}+n N_{2}-\varkappa n} \\
+ & z^{\prime}\left(1-\bar{c}_{s}\right) \frac{n_{a}}{N_{1}+n N_{2}-\varkappa n} \\
= & z^{\prime} \frac{N_{1}+n N_{2}-\bar{\alpha}_{s} n_{a}-\varkappa n}{N_{1}+n N_{2}-\varkappa n} . \\
= & z^{\prime} \frac{N_{1}+n N_{2}-\beta_{s}}{N_{1}+n N_{2}-\varkappa n} .
\end{aligned}
$$

Das 3. Segment hat ebenso viel Einstellmöglichkeiten wie das 2. Segment usw.

Das ganze 2. Molekül hat also

$$
z\left(N_{1}+n N_{2}-\beta_{s}\right) \cdot z^{\prime(x-1)}\left[\frac{N_{1}+n N_{2}-\beta_{s}}{N_{1}+n N_{2}-x n}\right]^{(x-1)}
$$

Anordnungsmöglichkeiten.

* In der vorangehenden Arbeit ${ }^{1}$ wurde die gegenseitige Behinderung von Zylindermolekülen in der Weise berechnet, daß das Molekül um seinen Schwerpunkt gedreht wurde, während wir hier das Segment um einen seiner Endpunkte drehen. Wie man sich leicht überzeugt, wenn man die geometrische Ableitung (Abschn. II der vorangegangenen Arbeit) unter dieser etwas anderen Voraussetzung durchrechnet, ist das ohne Einfluß auf das Ergebnis, da hierbei zwar $n_{a}$ größer, dafür aber $\alpha$ um denselben Faktor kleiner wird. Es ist auch nichts anderes zu erwarten, da die Wahl des Drehpunktes nur den Weg der Rechnung betrifft, jedoch das Endergebnis, nämlich die Anzahl der Anordnungsmöglichkeiten, nicht beeinflussen kann. Wir dürfen daher bei der Auswertung von (12) das Ergebnis der vorangegangenen Arbeit ohne Einschränkung übernehmen.
Das 3. Molekül

hat offenbar

$z\left(N_{1}+n N_{2}-\beta_{s}\right) \cdot z^{\prime(x-1)}\left[\frac{N_{1}+n N_{2}-2 \beta_{s}}{N_{1}+n N_{2}-x n}\right]^{(x-1)}$

Anordnungsmöglichkeiten und so fort für das 4., 5.... Molekül.

$N_{2}$ Makromoleküle haben somit (wenn man berücksichtigt, daß $N_{2} \gg 1$ ist $t^{2}$ insgesamt

$$
\begin{gathered}
z^{N_{2}}\left(N_{1}+n N_{2}\right)\left(N_{1}+n N_{2}-\beta_{s}\right) . .\left(N_{1}+n N_{2}-N_{2} \beta_{s}\right) \\
\cdot z^{N_{2}(x-1)}\left[\frac{\left(N_{1}+n N_{2}\right)\left(N_{1}+n N_{2}-\beta_{s}\right) \ldots}{\left(N_{1}+n N_{2}\right)\left(N_{1}+n N_{2}-\varkappa n\right) . .}\right. \\
\left.\frac{\left(N_{1}+n N_{2}-N_{2} \beta_{s}\right)}{\left(N_{1}+n N_{2}-N_{2} \varkappa n\right)}\right]^{(x-1)}
\end{gathered}
$$

Anordnungsmöglichkeiten. Die thermodynamische Wahrscheinlichkeit $W_{m}$ der Lösung ist also (wenn man noch Vertauschungen der Makromoleküle untereinander herausdividiert: Fạktor $\mathrm{N}_{2}$ ! im Nenner) :

$$
\begin{gathered}
W_{m}=\frac{z^{N_{2}} \cdot \beta_{s} N_{2}}{N_{2} !} \cdot \frac{\left(\frac{N_{1}+n N_{2}}{\beta_{s}}\right) !}{\left(\frac{N_{1}+n N_{2}}{\beta_{s}}-N_{2}\right) !}\left[\frac{z^{\prime} N_{2} \cdot \beta_{s}{ }^{N_{2}}}{(\varkappa n)^{N_{2}}}\right. \\
\left.\cdot \frac{\left(\frac{N_{1}+n N_{2}}{\beta_{s}}\right) !}{\left(\frac{N_{1}+n N_{2}}{\beta_{s}}-N_{2}\right) !} \cdot \frac{\left(\frac{N_{1}+n N_{2}}{\varkappa n}-N_{2}\right) !}{\left(\frac{N_{1}+n N_{2}}{\varkappa n}\right) !}\right]^{(x-1)} \cdot(4)
\end{gathered}
$$

Bezeichnen, wir den 1. Faktor mit $W_{a}$, den zweiten Faktor (in der eckigen Klammer) mit $W_{b}$, so ist also

$$
W_{m}=W_{a} \cdot W_{b},
$$

und die Mischungsentropie ist

$$
S_{m}=k \ln W_{a}+k \ln W_{b} .
$$

Die Verdünnungsentropie ist

$\Delta s_{1}^{m}=R \frac{\partial \ln W_{a}}{\partial n_{1}}+R \frac{\partial \ln W_{b}}{\partial n_{1}} \equiv \Delta s_{a}+\Delta s_{b}$.

Aus einem Vergleich von (4) mit (II, 5) bis (II, 8) ergibt sich, daß

$$
\Delta s_{a}=R \frac{n_{2}}{n_{1}}\left[1+\frac{n n_{2}}{n_{1}}\left(\frac{\beta_{s}}{2 n}-n\right)\right]
$$

ist. Auf Grund der im Anhang durchgeführten Rechnung ist

$$
\Delta s_{b}=R\left(\frac{n_{2}}{n_{1}}\right)^{2} n \frac{(x-1)\left(\beta_{s}-x n\right)}{2 n} ;
$$


Faßt man (6), (7) und (8) zusammen, so erhält man

$$
\begin{aligned}
& \Delta s_{1}^{m}= \\
& R \frac{n_{2}}{n_{1}}
\end{aligned}\left[1+\frac{n n^{2}}{n_{1}}\left(\frac{\beta_{s}}{2 n}-1+\frac{(x-1)\left(\beta_{s}-* n\right)}{2 n}\right)\right] .
$$

Nach Berücksichtigung von (2) und (3) ergibt sich hieraus

$\Delta s_{1}^{m}=R \frac{n_{2}}{n_{1}}\left[1+\frac{n n_{2}}{n_{1}}\left(\frac{x n_{a s}}{2 n} \bar{a}_{s}+\frac{\varkappa}{2}-1\right)\right]$.

Hierbei ist

$$
\varphi^{\prime} \equiv n n_{2} / n_{1}
$$

das Volumverhältnis des gelösten Stoffes zum Lösungsmittel. Ferner ist

$$
n_{s}=n / x
$$

offenbar die Anzahl der Gitterpunkte, die ein Segment besetzt. Führen wir (10) und (11) in (9) ein, so erhalten wir schließlich

$$
\Delta s_{1}^{m}=R \frac{n_{2}}{n_{1}}\left[1+\varphi^{\prime}\left(\frac{n_{a s}}{2 n_{s}} \bar{a}_{s}+\frac{\varkappa}{2}-1\right)\right] .
$$

III. Verdünnungsentropie und osmotischer Druck in Abhängigkeit von den Abmessungen eines Segments

Vergleicht man die für ein biegsames Molekül gültige Gl. (12) mit der für starre Zylindermoleküle abgeleiteten (II, 10), so erkennt man, daß sie sich von dieser nur dadurch unterscheidet, daß in ihr an Stelle der Größen $n_{a}, \bar{\alpha}$ und $n$ die entsprechenden Größen für ein einzelnes Segment $n_{a s}, \bar{\alpha}_{s}$ und $n_{s}$ auftreten. Wir können daher, indem wir die geometrische Ableitung der vorigen Arbeit verwenden, gemäß den Gl. (II, 26), (II, 26 a) und (II, $26 \mathrm{~b}$ ) für bewegliche Fadenmoleküle folgende Gleichungen als gültig ansehen:

wobei

$$
\Delta s_{1}^{m}=R \frac{n_{2}}{n_{1}}\left[1+\varphi^{\prime} f\left(q_{s}\right)\right]
$$

$$
f\left(q_{s}\right)=\frac{\pi}{4} \frac{\left(q_{s}-1\right)^{2}}{q_{s}-1 / 3}+2 \frac{q_{s}+1 / 3}{q_{s}-1 / 3}-1
$$

ist. Ist $q_{s}>10$, so kann man den vereinfachten Ausdruck

$$
f\left(q_{s}\right)=\frac{\pi}{4} q_{s}=0,785 q_{s}
$$

verwenden. Hierbei ist $q_{s}$ das Verhältnis der Längszur Querabmessung eines einzelnen Segments.

Für den osmotischen Druck $p$ gilt analog (II, 28)

$$
p=\frac{R T c^{\prime}}{M_{2}} \cdot\left(1+\frac{c^{\prime} f\left(q_{s}\right)}{1000 \underline{Q}}\right),
$$

wobei $c^{\prime}$ die Konzentration in $\mathrm{g} / l$ Lösungsmittel, $\mathrm{M}_{2}$ das Molekulargewicht des gelösten Stoffes und o dessen Dichte ist. Will man aus osmotischen Messungen die Abmessungen eines Segments ermitteln, so verwendet man analog (II, 29) die Gleichung

$$
f\left(q_{s}\right)=\frac{1000 \varrho}{c^{\prime}}\left[\frac{p / c^{\prime}}{\lim _{c=0}\left(p / c^{\prime}\right)}-1\right] .
$$

Aus dem so gefundenen $f\left(q_{s}\right)$-Wert ist dann nach (13a) oder (13b) $q_{s}$ zu berechnen. Für kleine $f\left(q_{s}\right)$-Werte ist $q_{s}$ aus II, Abb. 4, zu entnehmen.

Bei diesem Verfahren ist allerdings vorausgesetzt, daß der osmotische Druck allein durch die Mischungsentropie bestimmt ist. Wenn das nicht der Fall ist, dann kann Gl. (15) ebenso wie (14) auf keinen Fall verwendet werden. Es gilt aber die allgemeinere Gleichung

$$
f\left(q_{s}\right)=\frac{1000 \varrho}{c^{\prime}}\left[\frac{\Delta s_{1}^{m}}{\Delta s_{1}^{*}}-1\right],
$$

wobei $\Delta s_{1}^{*}$ die Verdünnungsentropie ist, die man nach dem Idealgesetz berechnet: $d$. h. es ist

$$
\Delta s_{1}^{*} \equiv R \frac{n_{2}}{n_{1}} .
$$

Schließlich ist noch der Einfluß der Solvatation zu berücksichtigen. Wird das Volumen, welches ein Molekül beansprucht, um den Faktor $\Phi$ vergrößert, so ist, wie in der vorangegangenen Arbeit gezeigt wurde, an Stelle von (15)

$$
f\left(q_{s}\right)=\frac{1000 \varrho}{c^{\prime} \sqrt{\Phi}}\left[\frac{p / c^{\prime}}{\lim \left(p / c^{\prime}\right)}-1\right]
$$

bzw. an Stelle von (16)

$$
f\left(q_{s}\right)=\frac{1000 \varrho}{c^{\prime} \sqrt{\Phi}}\left[\frac{\Delta s_{1}^{m}}{\Delta s_{1}^{*}}-1\right]
$$

zu setzen. Leider ist es sehr schwierig, über $\Phi$ eine sichere Angabe zu erhalten. Nimmt man an, daß jedes Grundmolekül des gelösten Stoffs 1 Lösungsmittelmolekül bindet, so ist $\Phi=2$ und $\sqrt{\Phi}=1,4$. Umgibt sich das gelöste Molekül mit 
einer monomolekularen Solvatschicht, so ist $\Phi \approx 10$ und $\sqrt{\Phi} \approx 3$. Bei Lösungen mit stark exothermer Verdünnungswärme sind $u$. U. noch höhere Werte für $\Phi$ anzunehmen. Da jedoch in die Formeln nụ $\sqrt{\Phi}$ eingeht, wird das keinen allzugroßen Einfluß haben. Wir werden im folgenden einfach $\sqrt{\Phi}=2$ setzen und mit den dadurch erhaltenen Näherungsgleichungen

und

$$
f\left(q_{s}\right)=\frac{500 \varrho}{c^{\prime}}\left[\frac{p / c^{\prime}}{\lim \left(p / c^{\prime}\right)}-1\right]
$$

$$
f\left(q_{s}\right)=\frac{500 \varrho}{c^{\prime}}\left[\frac{\Delta s_{1}^{m}}{\Delta s_{1}^{*}}-1\right]
$$

arbeiten. Es sei darauf hingewiesen, daß ebenso wie in der vorigen Arbeit das Achsenverhältnis $\dot{q}_{s}$ auf ein „trockenes“ Segment bezogen ist.

Das osmotische Verhalten der beiden Grenzfälle des starren und des innerlich beweglichen Fadenmoleküls drückt sich in den abgeleiteten Gleichungen sehr einfach aus. Das starre Molekül besteht im ganzen nur aus einem Segment. Der für solche Stoffe gefundene $q$-Wert ist also das Achsenverhältnis des Gesamtmoleküls. Sind andrerseits die Grundmoleküle eines Kettenmoleküls frei gegeneinander beweglich, so findet man für $q_{s}$ die Abmessungen eines einzelnen Grundmoleküls, also einen Wert in der Nähe von 1. Dann ist $f\left(q_{s}\right)$ ungefähr gleich 3, und Gl. (13) stimmt mit der entsprechenden für Kugelmoleküle abgeleiteten Gleichung überein. Das chemische Potential des Lösungsmittels einer solchen athermischen Lösung $\left(\Delta h_{1}=0\right)$ ist dann

$$
\Delta \mu_{1}=-T \Delta s_{1}^{m}=R T \frac{n_{2}}{n_{1}}\left(1+3 \varphi^{\prime}\right)
$$

in Übereinstimmung mit Gl. $(\mathrm{I}, 43 \mathrm{c})^{7}$. Sind die Grundmoleküle von gleicher Größe wie die des Lösungsmittels, so vermindert sich noch der Faktor 3 , so daß sich die van t'H off sche Gleichung ergibt. Das stimmt mit den Rechnungen $\mathrm{M} \ddot{\mathrm{u}} \mathrm{n}$ $\mathrm{s}$ te $\mathrm{rs}^{4}$ überein.

Es sei noch kurz auf eine von mir früher aufgestellte Gleichung eingegangen, die den osmotischen

7 Die höheren Potenzen von $\varphi$ bzw. $\varphi^{\prime}$ treten in (20) nicht auf, da wir die Ableitung nicht bis zur 3. Näherung durchgeführt haben wie in der I. Arbeit. Über die Richtung, in welcher die 3. Näherung zu suchen ist, vergl. den Abschnitt III der vorangegangenen Arbeit.
Druck in Abhängigkeit von der Konzentration wiedergibt ${ }^{8}$. Sie lautet

$$
p=\frac{R T c}{M_{2}} \frac{1}{1-c \mathfrak{g}},
$$

wobei $\mathfrak{S}=(k / p)^{1 / v}$ ist. Die Größe $\mathfrak{g}$ hat die Dimension eines spezifischen Volumens (,,spezifisches Wirkungsvolumen") und wurde zunächst als das Volumen des gebundenen Lösungsmittels angesehen. Da sich jedoch bei dieser Interpretation ergäbe, daß von $1 \mathrm{~g}$ gelöster Substanz bis zu mehr als $100 \mathrm{ccm}$ gebunden sein würde, wurde die Auffassung, daß es sich hier um fest gebundenes Lösungsmittel handelt, später fallen gelassen und die Gleichung mehr als eine empirische Beziehung aufgefaßt. Sie gibt den Verlauf der $p / c(c)$ Kurve mit nur 2 empirischen Konstanten (die unabhängig vom Molekulargewicht sind) auffallend gut wieder und ist in zahlreichen Systemen nachgeprüft worden ${ }^{9}$. Wie diese Gleichung theoretisch zu interpretieren ist, bleibt vorläufig ungeklärt. Da sie bei Lösungen von Fadenmolekülen fast universell anwendbar ist, kann man annehmen, daß sie auch eine theoretisch faßbare Grundlage hat, die wohl allerdings erst gefunden werden kann, wenn die Thermodynamik fadenmolekularer Lösungen beśser als bisher durchgearbeitet sein wird. Sie scheint mit den Solvatationseffekten im Zusammenhang zu stehen, da sie hauptsächlich auf Lösungen mit negativer Verdünnungswärme anwendbar ist.

\section{Vergleich mit der Erfahrung}

Beim Vergleich der abgeleiteten Beziehungen mit Versuchsdaten ist darauf $\mathrm{zu}$ achten, daß jene nur für athermische Lösungen gelten. Dadurch wird ihre praktische Verwendbarkeit zur Zeit noch sehr eingeschränkt, da die Lösungen von Stoffen mit Fadenmolekülen in den meisten Fällen Verdünnungswärmen haben, welche auch in sehr kleinen Konsentrationen einen gegenüber der Verdünnungsarbeit $\Delta \mu_{1}$ nicht $\mathrm{zu}$ vernachlässigenden Betrag darstellen. Nur ein Fall eines innerhalb der Fehlergrenzen als athermisch anzusehenden Systems ist bekannt, nämlich Guttaperchalösungen, an welchen E. W olf $f^{11}$ durch osmotische Messungen die Verdünnungsarbeit $\Delta \mu_{1}$, die Verdünnungswärme $\Delta h_{1}$ und die Verdünnungsentropie $\Delta s_{1}$ bestimmt hat. Dieser Autor fand, daß $\Delta h_{1}=0$ und somit

$$
\Delta \mu_{1}=-T \Delta s_{1}
$$

${ }^{8}$ G. V. S ch u l z, Z. physik. Chem. (A) 158, 237 [1932]; 176, 317 [1936]; 184, 1 [1939].

• G. V.S c h u l z, Fortschr. Chem. Physik u. Techn. Makromol. Verbb. 2, 49 [1942].

11 E. W o lf f, Helv. Chim. Acta 23, 439 [1940]. 
ist. Unter diesen Umständen darf man annehmen, daß die gemessene Verdünnungsentropie gleich der theoretischen Verdünnungsentropie (genauer gleich dem ,Mischungsanteil“" der Verdünnungsentropie) ist, daß also

gilt.

$$
\Delta s_{1}=\Delta s_{1}^{m}
$$

\begin{tabular}{|c|c|c|c|c|c|}
\hline$c^{\prime}$ & $\begin{array}{c}T \Delta s_{1} \\
\left(\begin{array}{c}\text { exper. } \\
\text { cal Mol } \\
10^{2}\end{array}\right.\end{array}$ & $\begin{array}{c}T \Delta s_{1}{ }^{*} \\
\text { nach }(17)\end{array}$ & $\frac{\Delta s_{1}}{\Delta s_{1}{ }^{*}}$ & $\begin{array}{c}f(q s) \\
\text { nach } \\
(19 \mathrm{a})\end{array}$ & $\begin{array}{c}q s \text { nach } \\
(13 \mathrm{~b})\end{array}$ \\
\hline 25 & 11 & 7,5 & 1,95 & 20 & 25,5 \\
36 & 19 & 5,8 & 2,45 & 19 & 24,4 \\
\hline
\end{tabular}

Tab. 1. Athermische Lösung: Guttapercha in Toluol. $M_{2}=30000 ; \varrho=0,95 ; \Delta h_{1}=0$ (für beide Konzentrationen).

Die Messungen an Guttapercha sind in Tab. 1 zusammengestellt. Für das Achsenverhältnis des Segments ergibt sich 25. Da ein Isoprenrest in der Kette 4. C-Atome und senkrecht dazu eine Methylgruppe besitzt, kann man für das Achsenverhältnis eines Grundmoleküls etwa 2 setzen. Danach besteht ein Segment aus 12 Isoprenresten. sind die aus osmotischen Messungen berechneten Segmentlängen eher etwas zu hoch (wegen des unsicheren Solvatationsfaktors). Somit ist die Übereinstimmung zwischen dem osmotischen und dem viskosimetrischen Wert so gut, wie man nur erwarten kann. Der wahrscheinlichste Wert dürfte dazwischen, etwa bei 8 bis 10 Einheiten, liegen. Ein Guttaperchamolekül vom Molekulargewicht 30000 (Polymerisationsgrad 440) verhält sich also hydrodynamisch und thermodynamisch so, als ob es aus 50 Segmenten bestünde, die etwa 20 -mal so lang wie dick und gegeneinander völlig frei beweglich sind.

Die übrigen mir bekannten osmotischen Messungen sind nicht in der einfachen Weise interpretierbar wie das soeben behandelte Beispiel. Um so wichtiger ist es, daß die Auswertung der osmotischen Messungen an der athermischen Guttaperchalösung zu Ergebnissen führt, die mit den aus andersartigen Messungen gewonnenen gut übereinstimmen. Hierdurch hat man eine einigermaßen sichere Basis gewonnen, um ein Verständnis der jetzt zu besprechenden Systeme anzubahnen, deren Verdünnungswärme nicht vernachlässigt werden kann.

In Tab. 2 sind Messungen verschiedener Autoren an Kautschuklösungen zusammengestellt. An

\begin{tabular}{|c|c|c|c|c|c|c|c|}
\hline$c^{\prime}$ & $\begin{array}{c}\Delta \mu_{1} \cdot 10^{2} \\
\text { (exper) }\end{array}$ & $\begin{array}{c}\Delta h_{1} \cdot 10^{2} \\
(\exp .)\end{array}$ & $\begin{array}{c}T \Delta s_{1} \cdot 10^{2} \\
\quad(\exp .)\end{array}$ & $\begin{array}{c}T \Delta s_{1}{ }^{*} \cdot 10^{2} \\
\text { nach }(17)\end{array}$ & $\frac{\Delta s_{1}}{\Delta s_{1}^{*}}$ & $\begin{array}{c}f\left(q_{s}\right) \\
\operatorname{nach}(19 \mathrm{a})\end{array}$ & $\begin{array}{c}q_{s} \\
\operatorname{nach}(13 \mathrm{~b})\end{array}$ \\
\hline \multicolumn{8}{|c|}{$M_{2}=150000 ;(\text { Gee u. Treloar })^{14}$} \\
\hline 13 & $-0,75$ & $+1,25 \pm 0,6$ & $+2,0 \pm 0,6$ & ca 0,4 & ca 5 & 146 & 186 \\
\hline 18 & $-0,4$ & $+1,8 \pm 0,4$ & $+3,3 \pm 0,4$ & ca 0,6 & ca 5,5 & 119 & 151 \\
\hline 25 & $-3,05$ & $+3,1 \pm 1,5$ & $+6,2 \pm 1,5$ & ca 0,8 & ca 7,7 & 127 & 162 \\
\hline \multicolumn{8}{|c|}{$\mathrm{M}_{2}=270000 ;(\text { K. H. M eyer, Wolff u. Boissonnas })^{15}$} \\
\hline 32,5 & $-7,8$ & $+2,0$ & +11 & $+0,74$ & 15 & 160 & 203 \\
\hline 44 & $-13,3$ & $+4,5$ & +19 & $+1,0$ & 19 & 153 & 194 \\
\hline
\end{tabular}

Tab. 2. Lösungen mit positiver Verdünnungswärme: Kautschuk in Benzol ( $\varrho=0,95)$; alles in cal/Mol.

Es ist interessant, diesen Wert mit dem zu vergleichen, den $\mathrm{W}$. und $\mathrm{H}$. $\mathrm{Ku} \mathrm{h} \mathrm{n}^{12}$ aus viskosimetrischen Messungen an Kautschukkohlenwasserstoffen ausrechnen. Sie finden für eine statistische Einheit (Segment) 5 Isoprenreste. Anscheinend liefert die $\mathrm{Ku} \mathrm{h} \mathrm{n}$ sche Berechnungsart allgemein etwas zu niedrige Werte für ein Segment, wie man aus einem Vergleich seiner Berechnungen für Nitrocellulose und der Sedimentationsmessungen Mosimanns ${ }^{13}$ entnehmen kann. Andrerseits

${ }^{12}$ W. K u h n u. H. K u h n, ebenda 26, 1394 [1943].

13 H. M o s i m a n n, ebenda 26, 61 [1943]. ihnen wurden durch Messung des osmotischen Druckes und seiner Temperaturabhängigkeit nebeneinander die Größen $\Delta \mu_{1}, \Delta h_{1}$ und $\Delta s_{1}$ bestimmt, die durch die Gleichung

$$
\Delta \mu_{1}=\Delta h_{1}-T \Delta s_{1}
$$

zusammenhängen. Wie man sieht, ist in den Kautschuklösungen $\Delta h_{1}$ positiv (endotherm), und zwar

${ }^{14}$ G. G e e u. L. R. G. Tr e l o a r, Trans. Faraday Soc. 38, 147 [1942].

15 K. H. Me y er, E. W olf $\mathrm{f}$. Ch. G. B o is s onn a s, Helv. Chim. Acta 23, 430 [1940]. 


\begin{tabular}{|c|c|c|c|c|c|}
\hline$c$ & $\Delta \mu_{1} \cdot 10^{2}$ & $\Delta h_{1} \cdot 10^{2}$ & $T \Delta s_{1} \cdot 10:$ & $T \Delta s_{1}^{*} \cdot 10^{*}$ & $\Delta s_{1} / \Delta s_{1}^{*}$ \\
\hline \multicolumn{6}{|c|}{ Polystyrol in Toluol; $M=90000$ (G.V.Schulz ${ }^{17}$ ) } \\
\hline $\begin{array}{r}9,9 \\
19,6 \\
29,0\end{array}$ & $\begin{array}{l}-1,19 \\
-3,12 \\
-5,57\end{array}$ & $\begin{array}{l}-0,47 \\
-1,66 \\
-3,44\end{array}$ & $\begin{array}{l}+0,72 \\
+1,46 \\
+2,18\end{array}$ & $\begin{array}{l}+0,76 \\
+1,41 \\
+2,10\end{array}$ & $\begin{array}{l}0,95 \\
1,114 \\
1,04\end{array}$ \\
\hline \multicolumn{6}{|c|}{ Nitrocellulose in Aceton; $\mathbf{M}=82000$ (G. V. Schulz ${ }^{17}$ ) } \\
\hline $\begin{array}{l}10 \\
20 \\
30\end{array}$ & $\begin{array}{l}-1,08 \\
-3,07 \\
-5,45\end{array}$ & $\begin{array}{l}-0.45 \\
-2,(16 \\
-3,93\end{array}$ & $\begin{array}{l}+0,36 \\
+1,(1) \\
+1,52\end{array}$ & $\begin{array}{r}+0.54 \\
+1,08 \\
+1, n 2\end{array}$ & $\begin{array}{l}1,17 \\
0,94 \\
0,94\end{array}$ \\
\hline \multicolumn{6}{|c|}{ Nitrocellulose in Cyclohexanon); $\mathrm{M}=66000$ (Meyer u. Boissonnas $\left.{ }^{1 \mathrm{~s}}\right)$} \\
\hline $\begin{array}{l}16,1 \\
31,5 \\
58,0\end{array}$ & $\begin{array}{l}-4,05 \\
-13,0 \\
-38,0\end{array}$ & $\begin{array}{l}1,5 \pm 1 \\
-9,0 \pm 2 \\
-17 \pm 3\end{array}$ & $\begin{array}{l}+2,6 \pm 1 \\
+\quad 4,0 \pm 2 \\
+21 \pm 3\end{array}$ & $\begin{array}{r}+1,5 \\
+2,9 \\
+5,4\end{array}$ & $\begin{array}{l}1,7 \pm 0,7 \\
1,4 \pm 0,7 \\
3,5 \pm 0,6\end{array}$ \\
\hline \multicolumn{6}{|c|}{ Acetylcellulose in Tetrachloräthan; $\mathrm{M}=42000$ (Hagger u. van der $\mathrm{Wyk}^{19}$ ) } \\
\hline $\begin{array}{r}6,7 \\
14,6 \\
35,4 \\
67,5\end{array}$ & $\begin{array}{l}-1,4 \\
-\quad 3,8 \\
-15,2 \\
-40,3\end{array}$ & $\begin{array}{l}-0,6 \\
-\quad 1,5 \\
-15,5 \\
-44,0\end{array}$ & $\begin{array}{l}+0,8 \\
+2,3 \\
+0,3 \\
-0,7\end{array}$ & $\begin{array}{l}+\quad 1,0 \\
+\quad 2,2 \\
+\quad 5,4 \\
+11\end{array}$ & $\begin{array}{l}0,8 \\
1,05 \\
0,05 \tilde{5} \\
-\end{array}$ \\
\hline
\end{tabular}

Tab. 3. Lösungen mit negativer Verdünnungswärme.

ist der Betrag der Verdünnungswärme so hoch, daß er nebèn den anderen Größen in (23) nicht vernachlässigt werden kann. Es erhebt sich daraufhin die Frage, ob man auch in solchen Fällen die differentiale Verdünnungsentropie als identisch mit der differentialen Mischungsentropie $\Delta s_{1}^{m}$ ansehen kann, so daß die oben abgeleiteten Gleichungen auf sie anwendbar sind.

Betrachtet man die 7. und 8. Spalte der Tab. 2, so erkennt man, daß in den Kautschuklösungen $f\left(q_{s}\right)$ und damit auch $q_{s}$ wesentlich höher ist als bei Guttapercha. Nimmt man für das Grundmolekül des Kautschuks wieder ein Achsenverhältnis von 2 an, so erhält man für das Segment eine Länge von etwa 100 Grundmolekülen. Wenn Kautschuk auch nicht mit Guttapercha identisch ist, so ist doch für die Steifheit dieser beiden Molekülarten keineswegs ein Unterschied von einer Größenordnung anzunehmen, zumal sich die beiden Kohlenwasserstoffe viskosimetrisch kaum unterscheiden ${ }^{16}$. Hieraus ist $\mathrm{zu}$ schließen, daß das experimentell bestimmte $\Delta s_{1}$ nicht identisch mit $\Delta s_{1}^{m}$ ist.

Das wird noch deutlicher, wenn wir jetzt die in

16 Vergl. H. S t a u d ing e r, Die hochmolekularen organ. Verbindungen, Springer Berlin 1932.

17 G. V. S c h u l z, Z. Elektrochem. angew. physik. Chem. 45, 652 [1939].

18 K. H. M e y e r u. Ch. G. B o is s o n n a s, Helv. Chim. Acta 20, 783 [1937].

19 O. H a g g e r u. A. J. A. v a n d e r W y k, Helv. Chim. Acta 23, 484 [1940].
Tab. 3 zusammengestellten Lösungen mit negativer (exothermer) Verdünnungswärme betrachten. In allen diesen Systemen ist in Konzentrationen bis $\mathrm{zu}$ etwa $3 \%$ (wo bereits starke Abweichungen vom Idealgesetz auftreten) $\Delta s_{1}$ annähernd gleich dem Idealwert, wie am deutlichsten die letzte Spalte der Tabelle zeigt. Nach den hier abgeleiteten Gleichungen müßte man daraus schließen, daß $q_{s}$ ungefähr gleich 1 ist, daß also die Moleküle des Polystyrols und der beiden Cellulosederivate eine sehr große innere Beweglichkeit besitzen. Aus ihrem viskosimetrischen Verhalten ${ }^{12}$ und bei der Nitrocellulose auch aus der Sedimentationsgeschwindigkeit in der Ultrazentrifuge ${ }^{13}$ geht jedoch hervor, daß sie verhältnismäßig starr sind, auf jeden Fall beträchtlich starrer als Kautschukmoleküle. Man muß daher annehmen, daß sich in diesen Lösungen der Mischungsentropie noch ein anderer Entropiebetrag überlagert, derart, daß $\Delta s_{1}$ kleiner ist als $\Delta s_{1}^{m}$.

Man ist versucht, diesen Befund zu verallgemeinern und folgende Beziehungen zwischen Verdünnungswärme und Verdünnungsentropie zu formulieren ${ }^{20}$.

20 Die Beziehungen (24) erinnern an Zusammenhänge zwischen Aktivierungsenergie und Aktionskonstante, die sich ergeben, wenn man die gleiche Reaktion in verschiedenen Lösungsmitteln ablaufen läßt. Vergl. Fa i r cloug h u. H in s h e l w o o d, J. Chem. Soc. [London] 1937, 538; G. V.S c h u l z, A. D i n gl in g e r u. E. Hu s e m a n n, Z. physik. Chem. (B) 43, 385 [1939]. 


$$
\left.\begin{array}{lll}
\Delta s_{1}>\Delta s_{1}^{m}, & \text { wenn } & \Delta h_{1}>0 \\
\Delta s_{1}=\Delta s_{1}^{m}, & \text { wenn } & \Delta h_{1}=0 \\
\Delta s_{1}<\Delta s_{1}^{m}, & \text { wenn } & \Delta h_{1}<0
\end{array}\right\} .
$$

Selbstverständlich reicht das bisher bekannte Material nicht dazu aus, um die Beziehungen (24) sicher zu fundieren, aber sie geben vielieicht einen Hinweis dafür, in welcher Richtung die weitere Klärung der thermodynamischen Verhältnisse makromolekularer Lösungen zu suchen ist. Jedenfalls ist es notwendig, bei einer theoretischen Untersuchung auf die ganz allgemeinen, in den Gl. (I,21) bis (I,23) formulierten Zusammenhänge zurückzugehen.

Experimentell wird es wichtig sein, denselben makromolekularen Stoff in verschiedenen Lösungsmitteln mit positiven und negativen Verdünnungswärmen zu untersuchen. Will man spe- ziell für einen makromolekulären Stoff durch osmotische Messungen $q_{s}$ ermitteln, so muß man dieses in einem Lösungsmittel ausführen, in welchem $\Delta h_{1}=0$ ist.

Leider wird diese Bedingung häufig von den auf diesem Gebiet theoretisch arbeitenden Forschern nicht beachtet $2,3,4,21$. So werden Beziehungen, welche für die Mischungsentropie abgeleitet sind, ohne weiteres auf den osmotischen Druck übertragen, wobei man die allgemeingültige Gl. (23) durch die nur unter speziellen Umständen geltende Gl. (21) ersetzt. Merkwürdigerweise wird das sogar bei Lösungen gemacht, welche auf Grund experimenteller Bestimmungen $\Delta h_{1}$-Werte haben, die von vergleichbarer Größe mit $\Delta \mu_{1}$ und $\Delta s_{1}$ sind. Es braucht eigentlich kaum darauf hingewiesen zu werden, daß dieses Verfahren jeder exakten Grundlage entbehrt, so daß damit erhaltene Ergebnisse auch dann, wenn sie mit Theorien der Verfasser übereinstimmen, keine reelle Bedeutung haben.

Anhang: Ableitung von $\Delta s_{b}$ aus $W_{b}$.

Nach Gl. (4) ist

$$
W_{b}=\left[\frac{z^{N_{2} \cdot \beta_{s} N_{2}}}{(\varkappa n)^{N_{2}}} \frac{\left(\frac{N_{1}+n N_{2}}{\beta_{s}}\right) !}{\left(\frac{N_{1}+n N_{2}}{\beta_{s}}-N_{2}\right) !} \cdot \frac{\left(\frac{N_{1}+n N_{2}}{\varkappa n}-N_{2}\right) !}{\left(\frac{N_{1}+n N_{2}}{\varkappa n}\right) !}\right] .
$$

Wir ersetzen nach $x-1=x^{\prime}$, dann erhalten wir durch Umformung nach der Sti r l in g schen Formel $(\mathrm{I}, 19)$ :

$$
\begin{aligned}
& \ln W_{b}=+\left\{N_{2} x^{\prime} \ln z^{\prime}+N_{2} x^{\prime} \ln \beta_{s}-N_{2} x \ln (x n)\right\}^{\circ} \\
& +\frac{x^{\prime}}{\beta_{s}}\left\{\left[N_{1}+n N_{2}\right] \ln \frac{N_{1}+n N_{2}}{\beta_{s}}-N_{1}-n N_{2}-\left[N_{1}+N_{2}\left(n-\beta_{s}\right)\right] \ln \frac{N_{1}+N_{2}\left(n-\beta_{s}\right)}{\beta}+N_{1}+N_{2}(n-\beta)\right\} \\
& -\frac{x^{\prime}}{x n}\left\{\left[N_{1}+n N_{2}\right] \ln \frac{N_{1}+n N_{2}}{n}-N_{1}-n N_{2}-\left[N_{1}+N_{2}(n-\varkappa n)\right] \ln \frac{N_{1}+n N_{2}(n-x n)}{n}\right. \\
& \left.+N_{1}+N_{2}(n-\varkappa n)\right\} .
\end{aligned}
$$

Wir führen jetzt ein: $N^{1}=N_{L} n_{1} ; N_{2}=N_{L} n_{2} ; R=N_{L} k$ und fassen alle Glieder, welche nicht $n_{1}$ enthalten, in den Ausdruck $f(n)_{2}$ zusammen. Dann erhalten wir

$$
\begin{aligned}
S_{b}=k \ln W_{b}=f\left(n_{2}\right)+\frac{R x^{\prime}}{\beta_{s}}\{( & \left.\left.n_{1}+n n_{2}\right) \ln \left(n_{1}+n n_{2}\right)-\left[n_{1}+n_{2}\left(n-\beta_{s}\right)\right] \ln \left[n_{1}+n_{2}\left(n-\beta_{s}\right)\right]\right\} \\
& -\frac{R x^{\prime}}{x n}\left\{\left(n_{1}+n n_{2}\right) \ln \left(n_{1}+n n_{2}\right)-\left[n_{1}+n_{2}(n-\varkappa n)\right] \ln \left[n_{1}+n_{2}(n-\varkappa n)\right]\right\} .
\end{aligned}
$$

Die Verdünnungsentropie erhalten wir durch Differentiation nach $n_{1}$. Es ist

$$
\begin{aligned}
\Delta s_{b} \equiv \partial S_{b} / \partial n_{1}=+\frac{R x^{\prime}}{\beta_{s}}\left\{\ln \left[n_{1}+n n_{2}\right]-\ln \left[n_{1}+n_{2}\left(n-\beta_{s}\right)\right]\right\} & \\
& \quad-\frac{R x^{\prime}}{\varkappa n}\left\{\ln \left[n_{1}+n n_{2}\right]-\ln \left[n_{1}+n_{2}(n-\varkappa n)\right]\right\}
\end{aligned}
$$

21 R. E. Pow el1, C. R. Cla r k u. H. Ey ring, J. Chem. Physies 9, 268 [1941]. 


$$
\begin{aligned}
& \Delta s_{b}=\frac{R x^{\prime}}{\beta_{s}} \ln \frac{1}{1-\frac{n_{2} \beta_{s}}{n_{1}+n n_{2}}}-\frac{R x^{\prime}}{x n} \ln \frac{1}{1-\frac{n_{2} x n}{n_{1}+n n_{2}}} \\
& \Delta s_{b}=\frac{R x^{\prime}}{\varkappa n} \ln \left[1-\frac{n_{2} \varkappa n}{n_{1}+n n_{2}}\right]-\frac{R x^{\prime}}{\beta_{s}}\left[\ln 1-\frac{n_{2} \beta_{s}}{n_{1}+n n_{2}}\right] .
\end{aligned}
$$

Entwickeln wir den Logarithmus in eine Reihe bis zum 2. Glied, so ergibt sich

$$
\begin{aligned}
\Delta s_{b} & =-R x^{\prime} \frac{n_{2}}{n_{1}+n n_{2}}+R x^{\prime} \frac{n_{2}}{n_{1}+n n_{2}}-\frac{R x^{\prime}}{2} \frac{n_{2}^{2} \varkappa n}{\left(n_{1}+n n_{2}\right)^{2}}+\frac{R x^{\prime}}{2} \frac{n_{2}^{2} \beta_{s}}{\left(n_{1}+n n_{2}\right)^{2}} \\
& =\frac{R x^{\prime}}{2} \frac{n_{2}^{2}}{\left(n_{1}+n n_{2}\right)^{2}}\left(\beta_{s}-\varkappa n\right) .
\end{aligned}
$$

Bei geringen Konzentrationen ist $n n_{2} \ll n_{1}$, und wir erhalten schließlich

$$
\Delta s_{b}=R\left(\frac{n_{2}}{n_{1}}\right)^{2} \frac{x^{\prime}\left(\beta_{s}-\varkappa n\right)}{2} .
$$

\section{Ein Beitrag zum Eiszeitproblem}

\section{Von Kurt Himpel}

Aus dem Physikalischen Institut der Universität Frankfurt a. M.

(Z. Naturforschg. 2 a, 419-427 [1947]; eingegangen am 1. April 1947)

Im ersten Teil werden kurz die entscheidenden Schwierigkeiten der beiden bekanntesten Versuche zur Erklärung der Eiszeiten, und zwar der Pol- bzw. Kontinentalverschiebungen (Epeirophorese) und der sog. astronomischen Theorie (Schwankungen der Schiefe der Ekliptik und der Erdbahnelemente) aufgezeigt. - Im zweiten Teil wird wahrscheinlich gemacht, daß eine schon 1921 vorgetragene Hypothese von S h a p le y, rach der die Sonne in der diluvialen Eiszeit eine von kosmischen Dunkelwolken verursachte schwache Veränderlichkeit ihrer Strahlung zeigte, nach neueren, in erster Linie astrophysikalischen Forschungsergebnissen eine brauchbare Erklärung zu liefern imstande ist, aus folgenden drei Gründen:

1. In allen dichteren kosmischen Dunkelwolken findet man unregelmäßige veränderliche Sterne eines ganz besonderen Typus; im Gegensatz $\mathrm{zu}$ fast allen übrigen Typen veränderlicher Sterne handelt es sich bei diesen Sternen um Zwergsterne, wie die Sonne oder noch schwächer.

2. Unser Sonnensystem befindet sich gegenwärtig innerhalb dunkler kosmischer Materie, und es ist höchstwahrscheinlich, daß es in naher Vergangenheit auch dichtere Teile von solchen Dunkelwolken durchquert hat.

3. Die Form der Dunkelwolken, ihre armförmigen, zirrus- oder zirrostratusähnlichen Anordnungen und Verästelungen lassen eine zwanglose Erklärung der mehrfachen Wiederholung der Eiszeiten zu; es wird hier also nicht nur die Eiszeit, sondern aus der gleichen Hauptursache auch deren Gliederung erklärt, was sonst nicht gelungen zu sein scheint. -

Die Mitwirkung anderer, in erster Linie astronomischer und geographischer Faktoren, letztere insbesondere in der älteren Erdgeschichte, ist durchaus möglich.

$\mathrm{V}$ or dreißig bis vierzig Jahren sah man der Lösung des Eiszeitproblems hoffnungsfreudiger entgegen als heute. Damals... erschien eine Hypothese über die Ursachen der Eiszeit nach der anderen... Heute ist man im allgemeinen bescheidener geworden und beschäftigt sich wieder mehr mit den Grundtatsachen ..." So lesen wir in dem ausgezeichneten Referat über den Stand des Problems bei Schw ar z b a c h ${ }^{1}$. Die Ursache für einen gewissen Pessimismus ist aber wohl nicht

1 M. Schwa r zbach, Das Problem der Eiszeit vom geologischen Standpunkt, Verlag F. Hirt, Breslau 1941. 